\section{P2-370 WEIGHT VARIATION OVER TIME AND ITS ASSOCIATION WITH TUBERCULOSIS TREATMENT OUTCOME: A LONGITUDINAL ANALYSIS}

doi:10.1136/jech.2011.142976l.1

${ }^{1} \mathrm{~A}$ Bernabe-Ortiz, ${ }^{1} \mathrm{C}$ Carcamo, ${ }^{2} \mathrm{~J}$ Sanchez, ${ }^{3} \mathrm{~J}$ Rios. ${ }^{1}$ Universidad Peruana Cayetano Heredia, Lima, Peru; ${ }^{2}$ US Naval Medical Research Unit No 6, Lima, Peru; ${ }^{3}$ Ministry of Health, Lima, Peru

Introduction Tuberculosis (TB) is a wasting disease. Weight variation has been proposed as a marker to predict TB therapy outcome. The goal of this study was to evaluate trends of patients' bodyweight over time depending upon TB treatment outcome.

Methods A retrospective cohort study with TB cases diagnosed from 2000 to 2006 was performed. Information from five public treatment facilities at San Juan de Miraflores, Lima, Peru was collected. Poor outcome was defined as failure or death during therapy, and compared to good outcome defined as cured. Longitudinal analysis using a marginal model was fitted using Generalised Estimating Equations to compare weight trends for patients with good and poor outcome, adjusting for age, sex, tuberculosis type, treatment scheme, BCG scar presence, HIV status and sputum variation during follow-up.

Results A total of 460 patients (55.4\% males) were included: 42 (9.1\%) had a poor outcome (17 failed and 25 died). Weight at baseline was not different between outcome groups. Interaction terms between outcome status and time were significant $(p=0.002)$ indicating that trends of bodyweight of patients with poor outcome completely differed of those with good outcome during follow-up. This divergence was observed from the first month of therapy (coefficient $-2.54, \mathrm{p}<0.001$ ) and was markedly different at fourth month of treatment (coefficient $-4.08, p=0.003$ ).

Conclusion Weight variation during tuberculosis therapy follow-up can predict treatment outcome. Patients with weigh loss during therapy or without gaining appropriate weight during treatment should be more closely followed as they are at risk of failure or death.

\section{P2-371 SOCIAL INEQUALITY IN HEALTH AMONG WOMEN}

doi:10.1136/jech.2011.142976l.2

C Senicato, M B de Azevedo Barros. * Universidade Estadual de Campinas, Campinas, Sao Paulo, Brazil

Introduction Increased involvement in the job market has added new responsibilities to traditional female roles, which are reflected in quality of life and health, but are manifested in a distinct fashion in this social segment. The present study assessed the health status and use of healthcare services of women between 20 and 59 years of age according to social strata based on level of schooling $(<9$ and 9 + years of study)

Methods A population-based cross-sectional study was carried out with conglomerate sampling. Five hundred eight women were analysed from a home survey carried out in Campinas, SP, Brazil in 2008. Prevalence values were estimated and prevalence ratios were adjusted using Poisson regression and considering weights related to the sampling design.

Results Women with a lower level of schooling had a poorer quality of life and greater prevalence values for hypertension ( $P R=1.63)$, circulatory problems $(\mathrm{PR}=1.92)$, dizziness $(\mathrm{PR}=1.92)$, headaches $(\mathrm{PR}=1.77)$, common mental disorders $(\mathrm{PR}=2.16)$ and a worse selfassessment of health $(\mathrm{PR}=2.44)$. No differences between groups were found regarding medical appointments in the previous 2 weeks, hospitalisations and surgeries in the previous year, self and clinical breast exams, Papanicolaou test and vaccine for rubella. Significant differences were found in the percentage of dental appointments and mammograms.

Conclusion Social inequalities were found in the prevalence of health problems, but no inequalities were found regarding various indicators of the use of healthcare services, which must be the fruit of the adequate structuring of the Brazilian public healthcare system in the city investigated.

\section{P2-372 RATES OF CHILDHOOD CANCER IN FEMALES REMAIN LOWER THAN EXPECTED IN DEVELOPING COUNTRIES}

doi:10.1136/jech.2011.142976l.3

${ }^{1,2} \mathrm{~S}$ Bhopal, ${ }^{1} \mathrm{~K}$ Mann, ${ }^{1} \mathrm{M}$ Pearce. ${ }^{1}$ Institute of Health and Society, Newcastle University, Newcastle Upon Tyne, UK; ${ }^{2}$ Newcastle Upon Tyne Hospitals NHS Foundation Trust, Newcastle Upon Tyne, UK

A decade ago it was reported that childhood cancer incidence was higher in boys than in girls in many countries, particularly in those with low gross domestic product and relatively high infant mortality rates. Reports from developing countries have since agreed that socio-economic or cultural rather than biological factors are likely to be responsible. The aim of this study was to investigate further the association between cancer registration sex ratios and economic, social and healthcare related factors using more recent data (1998-2002). Sex rate ratios for 63 countries were calculated from data published in Cancer Incidence in Five Continents Vol IX. An increased M:F sex rate ratio was significantly associated with decreasing life expectancy $(p=0.011)$, physician density $(p=0.029)$, per capita health expenditure $(p=0.005)$, GDP $(p=0.001)$ and female education (primary school enrolment sex ratio, $p=0.004$; secondary school enrolment sex ratio, $p=0.001$; adult literacy ratio, $p<0.001$ ) and increasing proportion of those living on less than US\$1 per day $(p=0.011)$. These associations persist when haematological malignancies (known to have higher incidence in boys) are excluded from analysis. Despite substantial investment in poverty reduction and healthcare over the intervening decade-and considerable improvements in health indicators - the previously observed disparity in girls being registered with cancer remains in countries with poor health system indicators and low female education rates, particularly in populations that are poor. We suggest that girls continue to go unregistered or undiagnosed for cancer and that incidence data, particularly in developing countries, should continue to be interpreted with caution.

\section{P2-373 A CLUSTER RANDOMISED CONTROLLED CLINICAL TRIAL TO ASSESS THE EFFICACY OF MULTIPLE MICRONUTRIENT SUPPLEMENTATIONS (IRON, VITAMINS A, C, FOLIC ACID AND ZINC) TO IMPROVE THE NUTRITIONAL AND HEALTH INDICATORS IN JEWISH AND BEDOUIN INFANTS IN SOUTHERN ISRAEL}

doi:10.1136/jech.2011.142976l.4

${ }^{1,2} \mathrm{~N}$ Bilenko, ${ }^{* 2,5}$ | Belmaker, ${ }^{2,4} \mathrm{H}$ Vardi, ${ }^{1,4} \mathrm{D}$ Fraser. ${ }^{1}$ Department of Epidemiology and Health Services Evaluation, Beer-Sheva, Israel; ${ }^{2}$ Regional Office of Ministry of Health, Southern Region, Beer-Sheva, Israel; ${ }^{3}$ Community Health Division, Ben-Gurion University of the Negev, Beer-Sheva, Israel; ${ }^{4}$ S. Daniel Abraham International Center for Health and Nutrition, Ben-Gurion University of the Negev, Beer-Sheva, Israel, ${ }^{5}$ Community Health Division, Ben-Gurion University of the Negev, Beer-Sheva, Israel

Background The immediate and long-term effects of nutritional deficiencies are increased incidence and severity of acute conditions, impaired rates of growth, and retarded cognitive development. An 